\title{
Efficacy and Safety of Linagliptin Co-Administered with Low-Dose Metformin Once Daily Versus High- Dose Metformin Twice Daily in Treatment-Naïve Patients with Type 2 Diabetes: a Double-Blind Randomized Trial
}

\author{
Linong Ji $\cdot$ Bernard Zinman $\cdot$ Sanjay Patel $\cdot$ Jinfeng Ji $\cdot$ \\ Zelie Bailes $\cdot$ Sandra Thiemann $\cdot$ Thomas Seck \\ To view enhanced content go to www.advancesintherapy.com \\ Received: November 26, 2014 / Published online: March 25, 2015 \\ (c) The Author(s) 2015. This article is published with open access at Springerlink.com
}

\section{ABSTRACT}

Introduction: The aim of this study was to investigate the efficacy and safety of linagliptin + low-dose (LD) metformin once

Trial registration: ClinicalTrials.gov number, NCT01438814.

Electronic supplementary material The online version of this article (doi:10.1007/s12325-015-0195-3) contains supplementary material, which is available to authorized users.

L. Ji (₫)

Department of Endocrinology and Metabolism, Peking University People's Hospital, Beijing, China e-mail: jilinong@gmail.com

\section{B. Zinman}

Lunenfeld-Tanenbaum Research Institute, Mount Sinai Hospital, University of Toronto, Toronto, ON, Canada

\section{S. Patel $\cdot$ Z. Bailes}

Boehringer Ingelheim Ltd, Bracknell, UK

J. Ji

Boehringer Ingelheim International Trading Co., Ltd, Shanghai, China

Present Address:

J. Ji

ICON Clinical Research, Beijing, China

S. Thiemann · T. Seck

Boehringer Ingelheim Pharma GmbH \& Co. KG, Ingelheim, Germany daily versus high-dose (HD) metformin twice daily in treatment-naïve patients with type 2 diabetes.

Methods: Patients $(n=689)$ were randomized (1:1) to double-blind treatment with linagliptin $5 \mathrm{mg}+\mathrm{LD}$ metformin (1000 mg) or HD metformin (2000 mg) for 14 weeks. Metformin was initiated at $500 \mathrm{mg} /$ day and up-titrated within 2 weeks; the dose then remained unchanged. The primary endpoint was change in glycated hemoglobin (HbA1c) from baseline to Week 14 in patients who tolerated a daily metformin dose of $\geq 1000 \mathrm{mg}$ after 2 weeks.

Results: At Week 14, HbA1c changed from a mean baseline of $8.0 \% \quad(64 \mathrm{mmol} / \mathrm{mol})$ by $-0.99 \%(-11 \mathrm{mmol} / \mathrm{mol})$ for linagliptin $+\mathrm{LD}$ metformin, and $-0.98 \%(-11 \mathrm{mmol} / \mathrm{mol})$ for HD metformin [treatment difference $-0.01 \%$ (95\% confidence interval $-0.13,0.12$ ) ( $0 \mathrm{mmol} / \mathrm{mol}), P=0.8924]$. The proportion of patients who achieved HbA1c $<7.0 \%$ $(53 \mathrm{mmol} / \mathrm{mol})$ without occurrence of moderate or severe gastrointestinal (GI) events (including abdominal pain, nausea, vomiting, diarrhea, and decreased appetite) was the same in both groups (51.3\% for both). Although the occurrence of moderate or severe GI events was 
similar, the linagliptin + LD metformin group had fewer mild GI events (18.5\% versus $24.3 \%$ ). The incidence of hypoglycemia was low in both groups.

Conclusion: Linagliptin + LD metformin combination showed similar efficacy and safety to HD metformin. This combination may be an alternative treatment option in patients who may have difficulty tolerating metformin doses $>1000 \mathrm{mg}$ /day.

Funding: Boehringer Ingelheim.

Keywords: Gastrointestinal events; Linagliptin; Metformin; Tolerability; Type 2 diabetes mellitus

\section{INTRODUCTION}

International guidelines for the treatment of type 2 diabetes mellitus (T2DM) collectively recommend metformin monotherapy, if not contraindicated, as first-line therapy [1-3]. These guidelines advocate personalizing targets for glycemic control, generally defined by a glycated hemoglobin (HbA1c) level $<7.0 \%$ $(53 \mathrm{mmol} / \mathrm{mol})[1,2]$ or $\leq 6.5 \%(48 \mathrm{mmol} / \mathrm{mol})$ [3]. However, treatment with metformin is associated with gastrointestinal (GI) events, including nausea, vomiting, diarrhea, abdominal pain, and loss of appetite [1, 4]. These symptoms limit its tolerability and may result in approximately 5\% of patients discontinuing metformin treatment $[5,6]$. GI disturbances may also reduce therapeutic adherence, potentially contributing to inadequate glycemic control. Therefore, metformin is initiated at a low dose (LD) and up-titrated to the maximum tolerated dose [1, 2].

Some guidelines advocate initial treatment with combination therapy in patients with baseline HbA1c levels of $\geq 9 \%(75 \mathrm{mmol} / \mathrm{mol})$
[1] or $\geq 7.5 \%$ (58 mmol/mol) [3]. This approach may also offer advantages when drug tolerability is a concern. For example, using lower doses of one or both drugs in the combination may minimize the adverse events (AEs) associated with the respective maximum dose monotherapies but without compromising the overall glucose-lowering efficacy [7].

Initial treatment with $\mathrm{LD}$ metformin plus another glucose-lowering drug could mitigate the tolerability issues associated with higher doses of metformin. LD metformin in combination with a dipeptidyl peptidase (DPP)-4 inhibitor is an attractive treatment option due to their complementary mechanisms of action which, together, target multiple pathophysiological defects of T2DM, with low additional risk for hypoglycemia and weight gain. Studies have shown that linagliptin plus metformin administered twice daily as add-on or initial combination therapy was effective and well tolerated for up to 2 years [8-10]. This combination did not increase the risk for hypoglycemia or weight gain, and GI tolerability was similar to that of metformin monotherapy. These results suggest this combination might be a valuable alternative treatment, especially for patients who do not tolerate higher or the maximum dose of metformin. Therefore, this study aimed to investigate the efficacy and safety of linagliptin $5 \mathrm{mg}$ in combination with $\mathrm{LD}$ metformin once daily versus high-dose (HD) metformin twice daily in treatment-naïve patients with T2DM.

\section{METHODS}

\section{Study Design and Patients}

This phase 4, randomized, double-blind, activecomparator controlled, parallel-group 
comparison study was performed at 88 trial centers in 13 countries (Belgium, Canada, China, Germany, Spain, India, Bangladesh, Guatemala, Mexico, Peru, Taiwan, Lebanon, and the Philippines; ClinicalTrials.gov number, NCT01438814). The study enrolled patients with a diagnosis of T2DM who were aged $\geq 18$ and $\leq 80$ years, with a body mass index (BMI) $\leq 45 \mathrm{~kg} / \mathrm{m}^{2}$, and $\mathrm{HbA} 1 \mathrm{c} \geq 7.0 \%$ $(53 \mathrm{mmol} / \mathrm{mol})$ and $\leq 10.0 \% \quad(86 \mathrm{mmol} / \mathrm{mol})$. Patients were drug-naïve, defined as an absence of any oral or injectable antihyperglycemic therapies for $\geq 12$ weeks before randomization.

Patients were ineligible if they had uncontrolled hyperglycemia [glucose level $\quad>240 \mathrm{mg} / \mathrm{dL} \quad(13.3 \mathrm{mmol} / \mathrm{l}) \quad$ during screening or placebo run-in after an overnight fast, confirmed by a second measurement]; acute coronary syndrome, stroke, or transient ischemic attack within 3 months before consent; indication of liver disease or impaired hepatic function; impaired renal function, defined as estimated glomerular filtration rate (eGFR) $\quad<60 \mathrm{~mL} / \mathrm{min} / 1.73 \mathrm{~m}^{2} \quad$ (determined during screening or placebo run-in); bariatric surgery within the past 2 years or other GI surgeries that induce chronic malabsorption; and a medical history of cancer or treatment for cancer within the last 5 years. Further exclusion criteria included blood dyscrasias or any disorder causing hemodialysis or unstable red blood cell; known history of pancreatitis or chronic pancreatitis; contraindications to metformin; treatment with anti-obesity drugs within 3 months before consent or any other treatment at the time of screening leading to unstable body weight; and treatment with systemic steroids at the time of informed consent or change in dosage of thyroid hormones within 6 weeks before informed consent or any other uncontrolled endocrine disorder except T2DM. Pre-menopausal women who were nursing, pregnant, or not practicing an acceptable method of birth control were also ineligible.

All patients gave written informed consent before participation. The trial protocol was approved by the independent ethics committees or institutional review boards of the participating centers. The study was carried out according to the principles of the Declaration of Helsinki and the International Conference on Harmonisation Guideline for Good Clinical Practice.

\section{Treatments}

Following screening, patients underwent a 2-week placebo run-in period. Treatment assignment was determined using an interactive voice/web response system and was stratified by $\mathrm{HbA} 1 \mathrm{c}$ at screening $[<8.5 \%$ $(69 \mathrm{mmol} / \mathrm{mol}) \quad$ or $\quad \geq 8.5 \% \quad(69 \mathrm{mmol} / \mathrm{mol})]$. Eligible patients were randomized (1:1) to double-blind treatment with either linagliptin $5 \mathrm{mg}$ and LD metformin once daily or HD metformin twice daily for 14 weeks, followed by a 1-week follow-up period. Metformin (immediate release formulation) was initiated at $500 \mathrm{mg} /$ day and was up-titrated within 2 weeks to a maximum daily dose of $1000 \mathrm{mg}$ in the LD group and a maximum daily dose of $2000 \mathrm{mg}$ in the HD group. During this 2-week period, metformin was up-titrated in multiples of $500 \mathrm{mg}$ every 3-4 days if, in the opinion of the investigator, no major GI or other events occurred. If these events occurred, metformin was down-titrated to the previous tolerated dose. For the following 12 weeks, the dose of metformin was unchanged.

Rescue therapy (antihyperglycemic drug selected by the investigator and taken in accordance with local prescribing information) 
was initiated if the following criteria were met: during the first 8 weeks, the patient had a fasting plasma glucose (FPG) level $>240 \mathrm{mg} / \mathrm{dL}$ $(13.3 \mathrm{mmol} / \mathrm{L})$ after an overnight fast; from Weeks 8 to 14, the patient had an FPG level of $>210 \mathrm{mg} / \mathrm{dL}(11.6 \mathrm{mmol} / \mathrm{L})$ after an overnight fast. To initiate rescue medication, these criteria had to be confirmed by two measurements, of which at least one was to have been performed after an overnight fast at the investigational site. If the patient's hyper- or hypoglycemia could not be controlled and the investigator anticipated no further effect from rescue therapy, the patient was discontinued from the trial. Glucagon-like peptide-1 analogs, DPP-4 inhibitors, and metformin were not allowed as rescue therapy.

\section{Endpoints}

The primary efficacy endpoint was the change from baseline in HbA1c after 14 weeks of treatment in patients who tolerated a daily metformin dose of $\geq 1000 \mathrm{mg}$ after 2 weeks. The first key secondary endpoint was a composite endpoint comprising the proportion of patients who achieved HbA1c $<7.0 \%(53 \mathrm{mmol} / \mathrm{mol})$ at Week 14 and without the occurrence of prespecified moderate or severe GI events; the second key secondary endpoint was the occurrence of pre-specified moderate or severe GI events during 14 weeks of treatment. The list of pre-specified GI events included AEs of abdominal pain, nausea, vomiting, diarrhea, and decreased appetite. Exact definitions of prespecified mild, moderate, and severe GI AEs are outlined in Table $\mathrm{S} 1$ in the electronic supplementary material (ESM). Other secondary endpoints included: change from baseline in FPG after 14 weeks; change from baseline in HbA1c over time; percentage of patients who achieved HbA1c $\leq 6.5 \%$
( $48 \mathrm{mmol} / \mathrm{mol}$ ) after 14 weeks and without the occurrence of pre-specified moderate or severe GI events; percentage of patients who achieved a $\geq 0.5 \%(6 \mathrm{mmol} / \mathrm{mol})$ reduction in $\mathrm{HbA} 1 \mathrm{c}$ after 14 weeks; composite endpoint comprising the percentage of patients who achieved a $\geq 0.5 \%$ ( $6 \mathrm{mmol} / \mathrm{mol})$ reduction in HbA1c after 14 weeks without the occurrence of prespecified moderate or severe GI events; percentage of patients who achieved $a \geq 0.8 \%$ $(9 \mathrm{mmol} / \mathrm{mol})$ reduction in $\mathrm{HbA} 1 \mathrm{c}$ after 14 weeks; composite endpoint comprising the percentage of patients who achieved a $\geq 0.8 \%$ $(9 \mathrm{mmol} / \mathrm{mol})$ reduction in HbA1c after 14 weeks without the occurrence of prespecified moderate or severe GI events; intensity (mild, moderate, or severe) of prespecified GI events during 14 weeks of treatment as assessed by investigators; intensity scores (based on visual analog scale) of pre-specified GI events during 14 weeks of treatment as assessed by patients; and change from baseline in body weight over time. Other efficacy endpoints included the percentage of patients who achieved HbA1c $<7.0 \%$ $(53 \mathrm{mmol} / \mathrm{mol}) \quad$ and $\mathrm{HbA} 1 \mathrm{c} \leq 6.5 \%$ ( $48 \mathrm{mmol} / \mathrm{mol}$ ) after 14 weeks, and the use of rescue therapy. Safety assessments included the frequency and intensity of AEs, including hypoglycemia and AEs of special interest, and changes from baseline in vital signs, electrocardiogram, and clinical laboratory values.

\section{Statistical Analysis}

The primary endpoint was evaluated using an analysis of covariance (ANCOVA), with 'treatment' as a fixed classification effect and 'baseline HbA1c' as a linear covariate. This prespecified primary analysis was performed on the full analysis set $\left(\mathrm{FAS}_{1000 \mathrm{mg}}\right)$, which comprised 
all randomized and treated patients with a baseline HbA1c measurement and $\geq 1$ ontreatment HbA1c measurement, who tolerated a daily metformin dose of $\geq 1000 \mathrm{mg}$ at the end of the titration phase. A last observation carried forward (LOCF) approach was used to replace missing data. Non-inferiority (with a margin of $0.35 \%$ ) followed by superiority for the primary endpoint in a hierarchical sequence was used to test for the treatment effect of linagliptin $+\mathrm{LD}$ metformin compared with HD metformin. The overall significance level was 5\%. Sensitivity analyses were performed to assess the impact of missing data, important protocol violations, and premature discontinuation of trial medication on the primary endpoint. Sensitivity analyses utilized the FAS (LOCF), FAS $_{1000 \mathrm{mg}}$-completers (LOCF), and per-protocol set (PPS) $1000 \mathrm{mg}$ (LOCF) using the same model as for the primary analysis. Pre-specified subgroup analyses for the primary endpoint based on FAS $_{1000 m g}$ were performed on several baseline variables including race, ethnicity, geographical region, baseline $\mathrm{HbA1c}$, age, gender, time since diagnosis of diabetes, baseline BMI, and renal function. For the first key secondary endpoint, the non-completers considered failure (NCF) approach was used (e.g., patients who did not complete 14 weeks were counted as having not achieved an HbA1c of $<7 \%$ and no pre-specified GI AE). For the second key secondary endpoint, original results were used [original data as collected, with no imputation or exclusions (e.g., due to rescue therapy or repeat values)]. A logistic regression model with treatment and continuous baseline HbA1c was fitted on the binary response variable to calculate the estimate of the odds ratio (OR) of the two treatment groups. The remaining secondary endpoints were exploratory. Safety data were analyzed using descriptive statistics and were based on the treated set (TS), defined as all patients treated with $\geq 1$ dose of randomized study medication. AEs were coded using the Medical Dictionary for Drug Affairs (MedDRA) version 15.0 and are presented by system organ class and preferred term. AEs of special interest were analyzed using standardized MedDRA queries (SMQs; narrow versions) and included hypersensitivity reactions, skin lesions, hepatic AEs, renal AEs, and pancreatitis.

\section{RESULTS}

\section{Patient Disposition, Demographics, and Baseline Clinical Characteristics}

The first patient was enrolled on November 24, 2011 and the last patient visit occurred on March 5, 2013. Patients $(n=689)$ were randomized to linagliptin + LD metformin $(n=344)$ or HD metformin $(n=345)$; all patients received $\geq 1$ dose of study drug (TS; Fig. 1). Overall, 661 (95.9\%) patients completed the trial and $28(4.1 \%)$ patients $(n=14$ per group) prematurely discontinued. The most common reasons for discontinuation were 'lost to follow-up' $(1.2 \%)$ and 'refusal to continue trial medication' (1.2\%) in the linagliptin $+\mathrm{LD}$ metformin group, and 'other' reasons $(1.2 \%)$ in the HD metformin group. Of the patients in the FAS, 42 patients $(12.4 \%)$ in the linagliptin + LD metformin group were excluded from the FAS $_{1000 m g}$ as they did not tolerate a daily metformin dose of $1000 \mathrm{mg}$ after 2 weeks; by contrast, no patients were excluded in the HD metformin group. The imbalance in the exclusion of patients from the $\mathrm{FAS}_{1000 \mathrm{mg}}$ was due to the design of this study, which specified different rules of discontinuation for the two groups. During the titration phase, patients who were unable to tolerate the starting dose of metformin $(500 \mathrm{mg} /$ day; both treatment 


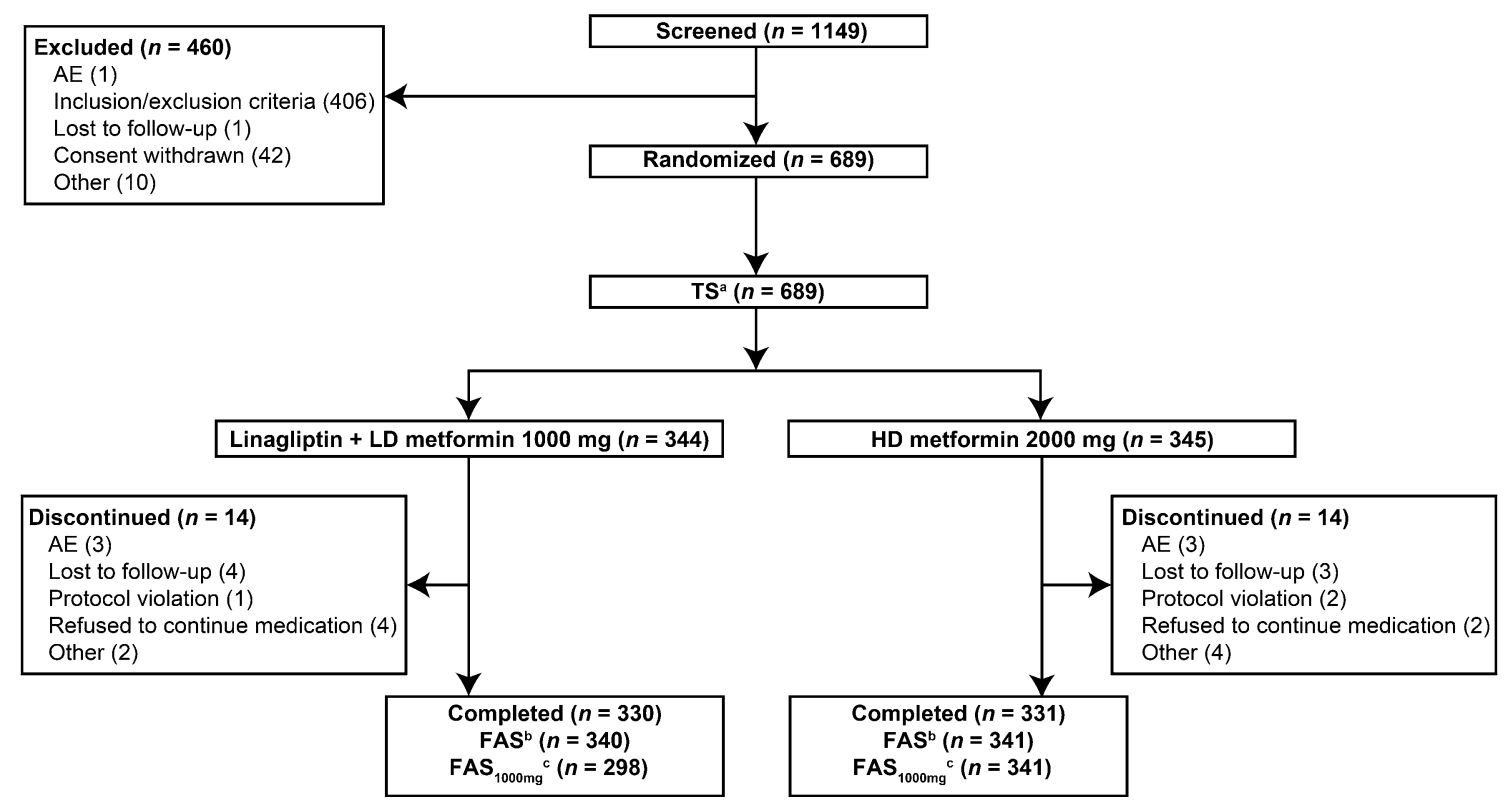

Fig. 1 Patient disposition. ${ }^{\text {aAll }}$ patients who were treated with $\geq 1$ dose of study medication. ${ }^{\mathrm{b}}$ All patients who had a baseline and $\geq 1$ on-treatment HbAlc measurement. ${ }^{c}$ All patients who had a baseline and $\geq 1$ on-treatment HbAlc

groups) or tolerate metformin $1000 \mathrm{mg} /$ day (HD metformin group only) were withdrawn from the trial. The FAS $_{1000 m g}$ consisted of 639 patients: linagliptin + LD metformin, $n=298$; HD metformin, $n=341$.

Demographic and clinical characteristics were similar between treatment groups (Table 1). The overall mean (SD) age, BMI, and HbA1c at baseline were 53.0 (10.7) years, $29.0(5.6) \mathrm{kg} / \mathrm{m}^{2}$, and $8.0(0.9) \% \quad[64$ (10) $\mathrm{mmol} / \mathrm{mol}]$, respectively. Just under half of patients had diabetes for $\leq 1$ year (47.3\%). The study population consisted primarily of Asian (47.6\%), American Indian/Alaska Native (26.7\%), and White patients (25.3\%).

Based on the TS, the mean (SD) metformin dose at Week 2 in the linagliptin $+\mathrm{LD}$ metformin group was $940.8(161.7) \mathrm{mg}$ /day (median $1000 \mathrm{mg} /$ day), with $88.2 \%$ of patients receiving $1000 \mathrm{mg} /$ day and $11.8 \%$ receiving $500 \mathrm{mg} /$ day. The mean (SD) metformin dose at Week 2 in the HD metformin group was measurement, who tolerated a daily metformin dose of $\geq 1000 \mathrm{mg}$ at the end of the titration phase. $A E$ adverse event, $F A S$ full analysis set, $H b A 1 c$ glycated hemoglobin, $H D$ high dose, $L D$ low dose, $T S$ treated set

1798.5 (347.6) mg/day (median $2000 \mathrm{mg} /$ day), with $71.8 \%$ of patients receiving $2000 \mathrm{mg} /$ day, $16.2 \%$ receiving $1500 \mathrm{mg} / \mathrm{day}$, and $12.1 \%$ receiving $1000 \mathrm{mg} /$ day.

\section{Efficacy Outcomes}

\section{Change in HbAlc and FPG}

Both groups showed clinically meaningful reductions in HbA1c levels at Week 14. The adjusted mean (SE) change in HbA1c from baseline was $\quad-0.99 \quad(0.05) \% \quad\left[\begin{array}{lll}-11 & \text { (1) }\end{array}\right.$ $\mathrm{mmol} / \mathrm{mol}]$ for linagliptin $+\mathrm{LD}$ metformin and $-0.98(0.04) \%\left[\begin{array}{lll}-11 & (0) \mathrm{mmol} / \mathrm{mol}] \text { for }\end{array}\right.$ HD metformin, resulting in a treatment difference of $-0.01(0.06) \%$ [95\% confidence interval (CI) $-0.13,0.12]$ [0 (1) $\mathrm{mmol} / \mathrm{mol}(95 \%$ CI $-1,1)] \quad(P=0.8924)$. Treatment with linagliptin + LD metformin was non-inferior $(P<0.0001)$, but not superior $(P=0.8924)$, to HD metformin. Figure $2 \mathrm{a}$ shows the change in HbA1c over time. 
Table 1 Baseline demographics and clinical characteristics

\begin{tabular}{|c|c|c|}
\hline & $\begin{array}{l}\text { Linagliptin } 5 \mathrm{mg}+\mathrm{LD} \\
\text { metformin } 1000 \mathrm{mg}\end{array}$ & $\begin{array}{l}\text { HD metformin } \\
2000 \mathrm{mg}\end{array}$ \\
\hline \multicolumn{3}{|l|}{ Demographics } \\
\hline Patients (TS), $n^{\mathrm{a}}$ & 344 & 345 \\
\hline Males, $n(\%)$ & $169(49.1)$ & $158(45.8)$ \\
\hline \multicolumn{3}{|l|}{ Race, $n(\%)$} \\
\hline American Indian/Alaska native & $85(24.7)$ & $99(28.7)$ \\
\hline Asian & $163(47.4)$ & $165(47.8)$ \\
\hline Black or African American & $0(0.0)$ & $3(0.9)$ \\
\hline White & $96(27.9)$ & $78(22.6)$ \\
\hline Age, years, mean $(\mathrm{SD})$ & $53.1(10.7)$ & $52.9(10.7)$ \\
\hline Body weight, kg, mean (SD) & $76.7(18.8)$ & $76.0(18.8)$ \\
\hline BMI, $\mathrm{kg} / \mathrm{m}^{2}$, mean $(\mathrm{SD})$ & $29.0(5.7)$ & $29.0(5.6)$ \\
\hline \multicolumn{3}{|l|}{ Renal function (eGFR) according to MDRD, $n$ (\%) } \\
\hline Normal $\left(\geq 90 \mathrm{~mL} / \mathrm{min} / 1.73 \mathrm{~m}^{2}\right)$ & $153(44.5)$ & $174(50.4)$ \\
\hline Mild impairment $\left(60\right.$ to $\left.<90 \mathrm{~mL} / \mathrm{min} / 1.73 \mathrm{~m}^{2}\right)$ & $176(51.2)$ & $169(49.0)$ \\
\hline Moderate impairment $\left(30\right.$ to $<60 \mathrm{~mL} / \mathrm{min} / 1.73 \mathrm{~m}^{2}$ ) & $15(4.4)$ & $2(0.6)$ \\
\hline \multicolumn{3}{|l|}{ Clinical characteristics } \\
\hline Patients $\left(\mathrm{FAS}_{1000 \mathrm{mg}}\right), n^{\mathrm{b}}$ & 298 & 341 \\
\hline HbA1c, \%, mean (SD) & $8.0(1.0)$ & $8.0(0.8)$ \\
\hline HbAlc, mmol/mol, mean (SD) & $64(10)$ & $64(9)$ \\
\hline $\mathrm{HbAlc}<8.5 \%(69 \mathrm{mmol} / \mathrm{mol}), n(\%)$ & $206(69.1)$ & $248(72.7)$ \\
\hline $\mathrm{FPG}, \mathrm{mg} / \mathrm{dL}$, mean $(\mathrm{SD})$ & $158.2(41.0)$ & $155.0(37.7)$ \\
\hline \multicolumn{3}{|l|}{ Duration of diabetes, $n(\%)$} \\
\hline$\leq 1$ year & $136(45.6)$ & $166(48.7)$ \\
\hline$>1$ to $\leq 5$ years & $103(34.6)$ & $112(32.8)$ \\
\hline$>5$ years & $59(19.8)$ & $63(18.5)$ \\
\hline
\end{tabular}

$B M I$ body mass index, $e G F R$ estimated glomerular filtration rate, $F P G$ fasting plasma glucose, $H b A 1 c$ glycated hemoglobin, $H D$ high dose, $L D$ low dose, $M D R D$ Modification of Diet in Renal Disease, $S D$ standard deviation

a TS—all patients who were treated with $\geq 1$ dose of study medication

b FAS $_{1000 \mathrm{mg}}$-all patients who had a baseline and $\geq 1$ on-treatment HbAlc measurement, who tolerated a daily metformin dose of $\geq 1000 \mathrm{mg}$ at the end of the titration phase

The sensitivity analyses were consistent with the results of the primary analysis: the comparator-adjusted mean (SE) change in
HbA1c from baseline at Week 14 was $-0.01(0.06) \% \quad(95 \% \quad$ CI $-0.13, \quad 0.12) \quad[0$ (1) $\mathrm{mmol} / \mathrm{mol}(95 \% \mathrm{CI}-1,1)]$ in the FAS 
(LOCF), $-0.01(0.07) \%(95 \% \mathrm{CI}-0.14,0.12)[0$ (1) $\mathrm{mmol} / \mathrm{mol}(95 \% \mathrm{CI}-2,1)]$ in the $\mathrm{FAS}_{1000 \mathrm{mg}^{-}}$ completers (LOCF), and -0.03 (0.06) \% (95\% CI $-0.15,0.10)[0(1) \mathrm{mmol} / \mathrm{mol}(95 \% \mathrm{CI}-2,1)]$ in the PPS $1000 \mathrm{mg}$ (LOCF).

The proportion of patients achieving HbA1c $<7.0 \%(53 \mathrm{mmol} / \mathrm{mol})$ at Week 14 was similar in both groups (linagliptin + LD metformin 56.7\%; HD metformin 56.3\%). Overall, 70.8\% of patients in the linagliptin $+\mathrm{LD}$ metformin group and $75.4 \%$ in the HD metformin group achieved $\mathrm{a} \geq 0.5 \%(6 \mathrm{mmol} / \mathrm{mol})$ reduction in HbA1c after 14 weeks. The proportion of patients achieving HbA1c $<6.5 \%$ $(48 \mathrm{mmol} / \mathrm{mol})$ and $\mathrm{a} \geq 0.8 \% \quad(9 \mathrm{mmol} / \mathrm{mol})$ reduction in HbA1c after 14 weeks was also similar (Table S2 in the ESM).

For both treatment arms, clinically relevant reductions in HbA1c were seen for all subgroups. None of the subgroups showed any notable differences between treatments, and no treatment by subgroup interactions was significant.

The adjusted mean (SE) change from baseline in FPG at Week 14 was $-24.5(1.5) \mathrm{mg} / \mathrm{dL}$ for linagliptin $+\mathrm{LD}$ metformin and $-26.6(1.4) \mathrm{mg} / \mathrm{dL}$ for $\mathrm{HD}$ metformin [comparator-adjusted mean (SE) change: $+2.0(2.1) \mathrm{mg} / \mathrm{dL}, 95 \% \mathrm{CI}-2.1,6.1$, $P=0.3352$ ] (Fig. 2b). Rescue medication was required by one patient in the linagliptin $+\mathrm{LD}$ metformin group and six patients in the HD metformin group.

\section{Safety and Tolerability}

\section{Pre-specified GI AEs}

The proportion of patients who had prespecified GI events of moderate or severe intensity during the treatment period was similar in both treatment groups (linagliptin + LD metformin $8.4 \%$; $\quad$ HD
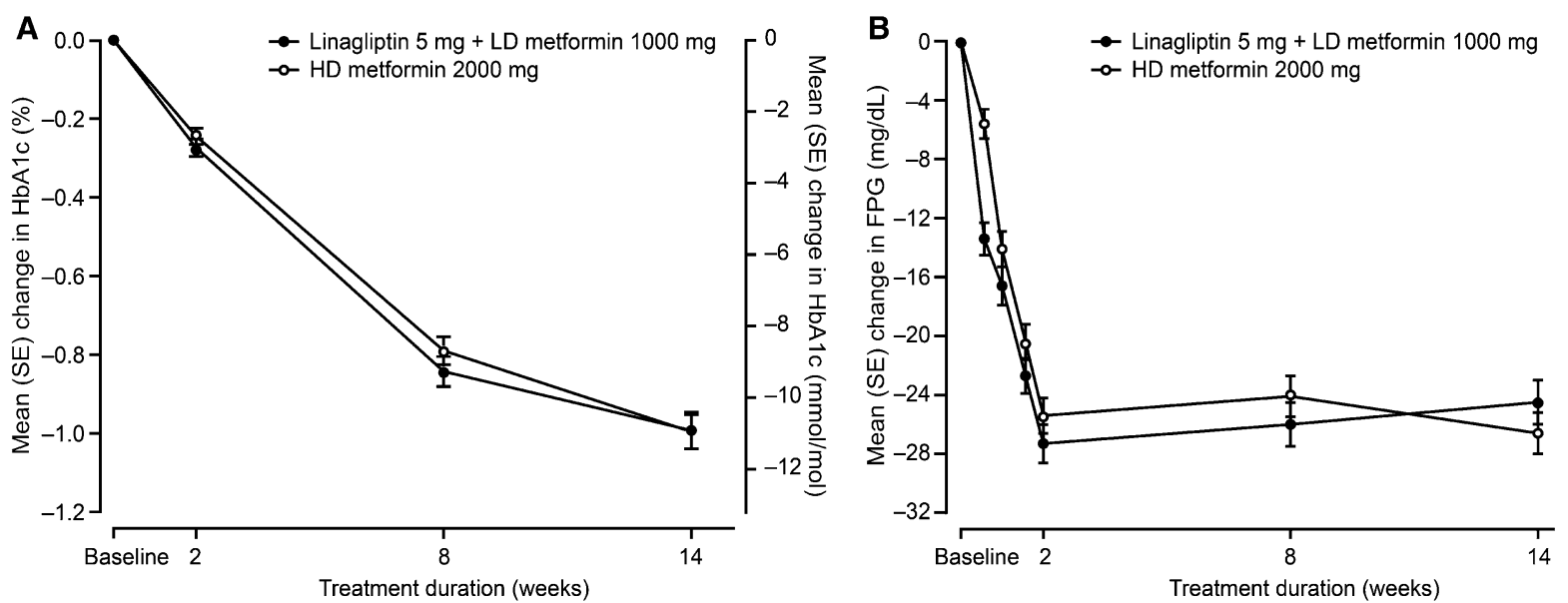

Fig. 2 Mean changes in HbAlc and FPG over time. a Adjusted mean change in HbAlc from baseline over time from the mixed model repeated measurements analysis ${ }^{\mathrm{a}}$ $\left(\mathrm{FAS}_{1000 \mathrm{mg}}\right.$; OC). $\mathbf{b}$ Adjusted $^{\mathrm{b}}$ mean change in FPG from baseline over time up to 14 weeks $\left(F_{1000 m g}\right.$; LOCF). ${ }^{a}$ Model includes treatment, continuous baseline $\mathrm{HbAlc}$ in addition to week repeated within patient, week by baseline $\mathrm{HbAlc}$ interaction, and week by treatment interaction. ${ }^{\mathrm{b}}$ ANCOVA model includes treatment, continuous baseline
HbAlc, and continuous baseline FPG. FAS $1000 \mathrm{mg}$-all patients who had a baseline and $\geq 1$ on-treatment HbAlc measurement, who tolerated a daily metformin dose of $\geq 1000 \mathrm{mg}$ at the end of the titration phase. $A N C O V A$ analysis of covariance, $F A S$ full analysis set, $F P G$ fasting plasma glucose, $H b A l c$ glycated hemoglobin, $H D$ high dose, $L D$ low dose, $L O C F$ last observation carried forward, $O C$ observed cases, $S E$ standard error 
metformin $8.2 \%$; OR 1.022, 95\% CI 0.6, 1.8, $P=0.9397$ ) (Table 2). The most frequently reported pre-specified GI events of moderate or severe intensity were diarrhea (linagliptin $+\mathrm{LD}$ metformin 6.4\%; HD metformin 5.6\%) and abdominal pain (linagliptin $+\mathrm{LD}$ metformin 2.3\%; HD metformin 3.2\%). The number of patients with pre-specified GI events of any intensity (mild, moderate, or severe) was lower in the linagliptin + LD metformin group compared with the HD metformin group. This difference was primarily due to fewer patients having mild GI events compared with the HD metformin group (18.5\% versus $24.3 \%$, respectively). The most frequently reported pre-specified GI events of mild intensity were diarrhea (linagliptin $+\mathrm{LD}$ metformin 12.1\%; HD metformin 15.8\%) and abdominal pain (linagliptin + LD metformin 3.4\%; HD metformin 6.2\%).

In both groups, most patients had the first GI event during the first 2 weeks (Figure S1 in the ESM). In the $\mathrm{FAS}_{1000 \mathrm{mg}}$, the adjusted mean (SE) visual analog scores for pre-specified GI event severity assessed by patients were similar in both groups [linagliptin + LD metformin 4.9 (0.2); HD metformin $4.4(0.2)]$.

\section{Change in Composite Endpoint}

The proportion of patients who achieved HbA1c $<7.0 \%(53 \mathrm{mmol} / \mathrm{mol})$ without occurrence of pre-specified moderate or severe GI events was the same in both groups (linagliptin $+\mathrm{LD}$ metformin 51.3\%; HD metformin 51.3\%; OR $0.960,95 \%$ CI $0.67,1.37, P=0.8201)$. The proportion of patients who achieved $\geq 0.5 \%$ $(6 \mathrm{mmol} / \mathrm{mol})$ reduction in $\mathrm{HbA} 1 \mathrm{c}$ without occurrence of pre-specified moderate or severe GI events after 14 weeks was similar (linagliptin + LD metformin 65.1\%; HD metformin 69.2\%). Patients who achieved HbA1c $\quad<6.5 \% \quad(48 \mathrm{mmol} / \mathrm{mol}) \quad$ without occurrence of pre-specified moderate or severe
GI events and who achieved $\geq 0.8 \%$ $(9 \mathrm{mmol} / \mathrm{mol})$ reduction in $\mathrm{HbA} 1 \mathrm{c}$ without occurrence of pre-specified moderate or severe GI events after 14 weeks were similar between the groups (Table S2 in the ESM).

\section{AEs and Hypoglycemia}

The overall frequency of any $\mathrm{AE}$ and drugrelated AEs was comparable between groups, and AEs leading to discontinuation of trial medication were low (Table 3). AEs of special interest were infrequent and no cases of pancreatic cancer were observed. One patient $(0.3 \%)$ treated with linagliptin + LD metformin and seven patients (2.0\%) treated with HD metformin had serious AEs requiring hospitalization. One patient treated with linagliptin + LD metformin had a serious $\mathrm{AE}$ that did not require hospitalization.

The highest frequency of AEs was reported for the system organ class GI disorders (linagliptin + LD metformin $33.7 \%$; HD metformin $35.1 \%$ ). GI AEs leading to discontinuation of trial medication were low and included only abdominal pain (linagliptin + LD metformin $0.6 \%$; HD metformin $0.3 \%$ ). Investigator-reported drugrelated GI AEs were reported in $17.7 \%$ of patients treated with linagliptin $+\mathrm{LD}$ metformin and $19.7 \%$ treated with HD metformin. The most common drug-related GI AEs ( $>2 \%$ in either group) were diarrhea $(9.3 \%$ versus $12.8 \%)$, abdominal pain (4.9\% versus $5.5 \%)$, and nausea (4.4\% versus $4.9 \%)$, respectively. Two patients reported serious GI AEs: acute pancreatitis in the linagliptin + LD metformin group, and abdominal pain and epiploic appendagitis (acute inflammation of an epiploic appendix) in the HD metformin group.

The incidence of investigator-reported hypoglycemia was low (linagliptin $+\mathrm{LD}$ metformin $0.6 \%$; HD metformin $1.7 \%$ ); no 
Table 2 Pre-specified GI events associated with metformin as assessed by the investigator

\begin{tabular}{|c|c|c|}
\hline & $\begin{array}{l}\text { Linagliptin } 5 \mathrm{mg}+\mathrm{LD} \\
\text { metformin } 1000 \mathrm{mg}\end{array}$ & $\begin{array}{l}\text { HD metformin } \\
2000 \mathrm{mg}\end{array}$ \\
\hline Patients $\left(\mathrm{FAS}_{1000 \mathrm{mg}}\right.$ ), $n^{\mathrm{a}}$ & 298 & 341 \\
\hline Patients with moderate or severe GI events, $n$ (\%) & $25(8.4)$ & $28(8.2)$ \\
\hline OR (95\% CI) versus metformin alone & $1.022(0.582,1.796)$ & \\
\hline Patients with any GI event, $n$ (\%) & $73(24.5)$ & $98(28.7)$ \\
\hline \multicolumn{3}{|l|}{ Patients by intensity, $n$ (\% of FAS $\left.{ }_{1000 \mathrm{mg}}\right)^{\mathrm{b}}$} \\
\hline Mild & $55(18.5)$ & $83(24.3)$ \\
\hline Moderate & $24(8.1)$ & $27(7.9)$ \\
\hline Severe & $4(1.3)$ & $1(0.3)$ \\
\hline Number of GI events, $n$ & 157 & 219 \\
\hline \multicolumn{3}{|l|}{ Events by intensity, $n$ (\% of total events) } \\
\hline Mild & $114(72.6)$ & $153(69.9)$ \\
\hline Moderate & $37(23.6)$ & $65(29.7)$ \\
\hline Severe & $6(3.8)$ & $1(0.5)$ \\
\hline \multicolumn{3}{|c|}{ Test of association between GI AE severity and treatment } \\
\hline Chi-square $P$ value & & 0.0314 \\
\hline Fishers exact $P$ value & & 0.0308 \\
\hline \multicolumn{3}{|l|}{ Patients with mild GI events, $n$ (\%) } \\
\hline Diarrhea & $36(12.1)$ & $54(15.8)$ \\
\hline Abdominal pain & $10(3.4)$ & $21(6.2)$ \\
\hline Nausea & $13(4.4)$ & $16(4.7)$ \\
\hline Vomiting & $5(1.7)$ & $6(1.8)$ \\
\hline Decreased appetite & $3(1.0)$ & $8(2.3)$ \\
\hline \multicolumn{3}{|l|}{ Patients with moderate or severe GI events, $n$ (\%) } \\
\hline Diarrhea & $19(6.4)$ & $19(5.6)$ \\
\hline Abdominal pain & $7(2.3)$ & $11(3.2)$ \\
\hline Nausea & $4(1.3)$ & $8(2.3)$ \\
\hline Vomiting & $5(1.7)$ & $1(0.3)$ \\
\hline Decreased appetite & $3(1.0)$ & $3(0.9)$ \\
\hline
\end{tabular}

Pre-specified GI events included AEs of abdominal pain, nausea, vomiting, diarrhea, and decreased appetite $A E$ adverse event, $C I$ confidence interval, $G I$ gastrointestinal, $H D$ high dose, $L D$ low dose, $O R$ odds ratio

${ }^{a}$ Full analysis set $\left(\mathrm{FAS}_{1000 \mathrm{mg}}\right.$ - -all patients who had a baseline and $\geq 1$ on-treatment HbAlc measurement, who tolerated a daily metformin dose of $\geq 1000 \mathrm{mg}$ at the end of the titration phase

b Patients could be counted in more than one category 
Table 3 Overall summary of AEs (TS)

\begin{tabular}{|c|c|c|}
\hline & $\begin{array}{l}\text { Linagliptin } 5 \mathrm{mg}+\mathrm{LD} \\
\text { metformin } 1000 \mathrm{mg}\end{array}$ & $\begin{array}{l}\text { HD metformin } \\
2000 \mathrm{mg}\end{array}$ \\
\hline Patients, $n$ & 344 & 345 \\
\hline Any AE, $n(\%)$ & $219(63.7)$ & $229(66.4)$ \\
\hline Severe AEs, $n$ (\%) & $12(3.5)$ & $9(2.6)$ \\
\hline Investigator-reported, drug-related AEs, $n$ (\%) & $71(20.6)$ & $80(23.2)$ \\
\hline AEs leading to discontinuation of trial medication, $n$ (\%) & $3(0.9)$ & $3(0.9)$ \\
\hline Serious AEs, $n(\%)$ & $2(0.6)$ & $7(2.0)$ \\
\hline \multicolumn{3}{|l|}{ Patients with AEs of special interest, $n$ (\%) } \\
\hline Hypersensitivity reactions & $1(0.3)$ & $1(0.3)$ \\
\hline Cutaneous skin lesions & $0(0.0)$ & $0(0.0)$ \\
\hline Hepatic AEs & $5(1.5)$ & $9(2.6)$ \\
\hline Renal AEs & $0(0.0)$ & $0(0.0)$ \\
\hline Pancreatitis & $1(0.3)$ & $0(0.0)$ \\
\hline
\end{tabular}

TS-all patients who were treated with $\geq 1$ dose of study medication

$A E$ adverse event, $H D$ high dose, $L D$ low dose, $T S$ treated set

severe hypoglycemic events requiring assistance were reported. Slight decreases in body weight were observed in both groups: the adjusted mean (SE) change from baseline after 14 weeks was $-0.44(0.14) \mathrm{kg}$ in the linagliptin $+\mathrm{LD}$ metformin group and $-1.05(0.13) \mathrm{kg}$ in the HD metformin group; the treatment difference was $0.62(0.19) \mathrm{kg} \quad(95 \% \quad \mathrm{CI} \quad 0.25, \quad 0.98$, $P=0.0011)$. There were no clinically relevant changes in vital signs or laboratory parameters in either group, and no patient had any cardiac or cerebrovascular event confirmed by the clinical event committee. No patient had any confirmed case of hospitalization due to heart failure.

\section{DISCUSSION}

Metformin is predominantly recommended as first-line therapy for the treatment of T2DM [1, 3], unless it is contraindicated. In clinical practice, metformin can be up-titrated to a maximum daily dose of $2550 \mathrm{mg}$ [11], with maximum efficacy achieved at $2000 \mathrm{mg}$ [5]. Metformin is well tolerated in the majority of patients; however, GI events are common and can occur in up to $30 \%$ of patients [5, 12-14]. These complications tend to occur soon after initiation of therapy $[1,15]$ and in particular when metformin is used at higher doses $[5,16]$. To minimize GI events and improve tolerability, guidelines recommend that metformin is initiated at a low dose and is gradually up-titrated to the maximum tolerated dose [1]. Although in many patients GI events tend to resolve upon continued treatment, or with a reduction in metformin dose, the symptoms can be severe enough to limit or complicate optimal dose titration and therefore negatively impact treatment adherence and longterm glycemic control [17].

This trial evaluated whether linagliptin $5 \mathrm{mg}+\mathrm{LD}$ metformin once daily is a potential 
alternative treatment to HD metformin twice daily with a focus on glycemic control and prespecified GI events in treatment-naïve patients with T2DM and insufficient glycemic control. The results show that linagliptin $+\mathrm{LD}$ metformin and HD metformin were equally effective and both substantially reduced HbA1c and FPG levels. Furthermore, more than half of patients in both arms achieved a clinically relevant HbA1c target of $<7.0 \%$ (53 mmol $/ \mathrm{mol})$. Since glycemic efficacy was similar in the combination group in the context of a substantially lower dose of metformin, linagliptin + LD metformin may be an appropriate treatment alternative, especially in patients who cannot tolerate higher doses of metformin. In contrast to the design of the present study, the effects of combination therapy with linagliptin + metformin have been investigated previously in the setting of equivalent doses of metformin monotherapy [8] and linagliptin monotherapy [18]. In the study by Haak et al. [8], initial combination of linagliptin (2.5 mg twice daily) with LD (500 mg twice daily) or HD (1000 mg twice daily) metformin achieved superior glucose-lowering efficacy compared with the equivalent doses of metformin monotherapy. In the study by Ross et al. [18], initial combination therapy with linagliptin (5 mg once daily) and metformin (up-titrated to $1000 \mathrm{mg}$ twice daily) significantly improved glycemic control compared with the equivalent dose of linagliptin monotherapy. Studies of other DPP-4 inhibitors have also shown significant clinical benefit when administered as initial combination therapy with metformin and compared with the equivalent dose of metformin monotherapy [19-23]. Therefore, the combination of linagliptin and metformin may be an appropriate choice if glycemic control is not achieved with metformin monotherapy.
Although controlling hyperglycemia is the most important property of glucose-lowering therapies, consideration should be given to overall tolerability [1]. This is a particular concern when using combination therapy due to the potential increase in AEs. When administering multiple oral antihyperglycemic drugs, one approach to avoid excessive AEs is to use a LD combination strategy [7, 24, 25]. This strategy could be especially useful in patients who do not tolerate maximum effective doses of one drug, such as metformin. In this study, about $30 \%$ of patients assigned to HD metformin could not achieve the maximum dose of $2000 \mathrm{mg}$. The proportion of patients who had pre-specified GI events of moderate or severe intensity during treatment was similar in both groups. However, the number of patients who had pre-specified GI events of any intensity was lower in the linagliptin + LD metformin group, mainly due to fewer patients experiencing mild GI events. Diarrhea and abdominal pain were the most commonly reported GI events, with lower incidences occurring with linagliptin + LD metformin. One possible explanation for the modest difference in GI events is that forced titration to maximal dose was not employed. GI events are more likely to occur soon after initiation of metformin [1, 15], an observation supported by this study. Previous studies have shown that even at equal metformin doses, patients receiving a DPP-4 inhibitor + metformin single-pill combination tend to experience fewer GI AEs compared with those receiving metformin alone [19, 26, 27]. The potential mechanism of this effect is currently unknown.

Despite significant improvements in glycemic control in both treatment groups, the incidence of hypoglycemia was low with no episodes of severe hypoglycemia requiring 
assistance. In addition, small decreases in body weight were seen in both groups.

This study was based on a relatively short duration of 14 weeks, which did not allow for assessments of long-term safety or durability of treatment efficacy. However, in a previous phase 3 study, the largest decrease in HbA1c occurred during the first 12 weeks of treatment for both the linagliptin + metformin combination group and the metformin monotherapy group. Furthermore, in this study the maximum effect for the change in FPG was achieved in both groups by Week 2, which suggests that most of the expected reduction in HbA1c was achieved by Week 14 . The combination of linagliptin plus metformin has also been shown to provide durable HbA1c reduction for up to 2 years [10]. This study did not assess whether patients receiving HD metformin and who were experiencing GI AEs would benefit from switching to linagliptin $+\mathrm{LD}$ metformin, which may have minimized the GI AEs without compromising the overall glucoselowering efficacy. In the present study, patients received immediate release metformin; however, extended release formulations of metformin are available which are associated with fewer GI AEs compared with immediate release metformin [12]. Other limitations include the lack of a forced titration scheme to up-titrate the dose of metformin and, because the study was conducted primarily (74\%) in Asian and American Indian/Alaska native patients, the possible underrepresentation of white patients could affect the applicability of these results to the wider T2DM patient population.

\section{CONCLUSION}

This study shows that treatment with linagliptin + LD metformin once daily provided similar glucose control to HD metformin twice daily in treatment-naïve patients with T2DM and insufficient glycemic control. The overall safety profile of linagliptin + LD metformin combination therapy was comparable to HD metformin monotherapy, with a low incidence of hypoglycemia in both groups. Protocol-prespecified moderate and severe GI events were similar for the two treatments, although the linagliptin + LD metformin group was associated with fewer mild GI events. Our findings suggest that linagliptin $+\mathrm{LD}$ metformin once daily may be an alternative treatment option in patients who cannot tolerate metformin doses $>1000 \mathrm{mg}$ /day.

\section{ACKNOWLEDGMENTS}

Sponsorship and article processing charges for this study were provided by Boehringer Ingelheim. All named authors meet the International Committee of Medical Journal Editors (ICMJE) criteria for authorship for this manuscript, take responsibility for the integrity of the work as a whole, and have given final approval for the version to be published. Medical writing assistance, supported financially by Boehringer Ingelheim, was provided by Paul MacCallum and Claire Stevens, of Envision Scientific Solutions during the preparation of this article.

Conflict of interest. L. Ji received a grant from Boehringer Ingelheim during the conduct of the study. B. Zinman received grants from Novo Nordisk, Boehringer Ingelheim, and Merck and received honoraria or consulting fees from Novo Nordisk, Boehringer Ingelheim, Eli Lilly, AstraZeneca, BMS, Jansen, Takeda, and Sanofi-Aventis. S. Patel is an employee of 
Boehringer Ingelheim. Z. Bailes is an employee of Boehringer Ingelheim. J. Ji was an employee of Boehringer Ingelheim at the time of the study, but is now an employee of ICON Clinical Research. S. Thiemann is an employee of Boehringer Ingelheim. T. Seck is an employee of Boehringer Ingelheim. No other potential conflicts of interest relevant to this article were reported.

\section{Compliance with ethics guidelines. All} patients gave written informed consent before participation. The trial protocol was approved by the independent ethics committees or institutional review boards of the participating centers. The study was carried out according to the principles of the Declaration of Helsinki and the International Conference on Harmonisation Guideline for Good Clinical Practice.

Open Access. This article is distributed under the terms of the Creative Commons Attribution Noncommercial License which permits any noncommercial use, distribution, and reproduction in any medium, provided the original author(s) and the source are credited.

\section{REFERENCES}

1. Inzucchi SE, Bergenstal RM, Buse JB, et al. Management of hyperglycemia in type 2 diabetes: a patient-centered approach: position statement of the American Diabetes Association (ADA) and the European Association for the Study of Diabetes (EASD). Diabetes Care. 2012;35:1364-79.

2. International Diabetes Federation. Clinical Guidelines Task Force. Global guideline for Type 2 diabetes. http://www.idf.org/sites/default/files/IDFGuideline-for-Type-2-Diabetes.pdf. Accessed July $28,2014$.

3. Garber AJ, Abrahamson MJ, Barzilay JI, et al. AACE comprehensive diabetes management algorithm 2013. Endocr Pract. 2013;19:327-36.
4. Aurobindo. Metformin summary of product characteristics. http://www.medicines.org.uk/emc/ medicine/23244/SPC. Accessed Feb 13, 2014.

5. Garber AJ, Duncan TG, Goodman AM, Mills DJ, Rohlf JL. Efficacy of metformin in type II diabetes: results of a double-blind, placebo-controlled, doseresponse trial. Am J Med. 1997;103:491-7.

6. Food and Drug Administration. Metformin hydrochloride tablets. http://www.fda.gov/ohrms/ dockets/dailys/02/May02/053102/800471e6.pdf. Accessed July 30, 2014.

7. Riddle M. Combining sulfonylureas and other oral agents. Am J Med. 2000;108(Suppl 6a):15S-22S.

8. Haak T, Meinicke T, Jones R, Weber S, von Eynatten $\mathrm{M}$, Woerle HJ. Initial combination of linagliptin and metformin improves glycaemic control in type 2 diabetes: a randomized, double-blind, placebocontrolled study. Diabetes Obes Metab. 2012;14:565-74.

9. Taskinen MR, Rosenstock J, Tamminen I, et al. Safety and efficacy of linagliptin as add-on therapy to metformin in patients with type 2 diabetes: a randomized, double-blind, placebo-controlled study. Diabetes Obes Metab. 2011;13:65-74.

10. Gomis R, Owens DR, Taskinen MR, et al. Long-term safety and efficacy of linagliptin as monotherapy or in combination with other oral glucose-lowering agents in 2121 subjects with type 2 diabetes: up to 2 years exposure in 24-week phase III trials followed by a 78-week open-label extension. Int J Clin Pract. 2012;66:731-40.

11. Bailey CJ, Turner RC. Metformin. N Engl J Med. 1996;334:574-9.

12. Blonde L, Dailey GE, Jabbour SA, Reasner CA, Mills DJ. Gastrointestinal tolerability of extended-release metformin tablets compared to immediate-release metformin tablets: results of a retrospective cohort study. Curr Med Res Opin. 2004;20:565-72.

13. Dandona P, Fonseca V, Mier A, Beckett AG. Diarrhea and metformin in a diabetic clinic. Diabetes Care. 1983;6:472-4.

14. Hoffmann IS, Roa M, Torrico F, Cubeddu LX. Ondansetron and metformin-induced gastrointestinal side effects. Am J Ther. 2003;10:447-51.

15. Haupt E, Knick B, Koschinsky T, Liebermeister H, Schneider J, Hirche H. Oral antidiabetic combination therapy with sulphonylureas and metformin. Diabete Metab. 1991;17:224-31. 
16. Hirst JA, Farmer AJ, Ali R, Roberts NW, Stevens RJ. Quantifying the effect of metformin treatment and dose on glycemic control. Diabetes Care. 2012;35:446-54.

17. Florez H, Luo J, Castillo-Florez S, et al. Impact of metformin-induced gastrointestinal symptoms on quality of life and adherence in patients with type 2 diabetes. Postgrad Med. 2010;122:112-20.

18. Ross $\mathrm{SA}$, Caballero AE, Del Prato $\mathrm{S}$, et al. Initial combination of linagliptin and metformin compared with linagliptin monotherapy in patients with newly diagnosed type 2 diabetes and marked hyperglycaemia: a randomized, doubleblind, active-controlled, parallel group, multinational clinical trial. Diabetes Obes Metab. 2015;17:136-44.

19. Bosi E, Dotta F, Jia Y, Goodman M. Vildagliptin plus metformin combination therapy provides superior glycaemic control to individual monotherapy in treatment-naive patients with type 2 diabetes mellitus. Diabetes Obes Metab. 2009;11:506-15.

20. Jadzinsky M, Pfützner A, Paz-Pacheco E, Xu Z, Allen E, Chen R. Saxagliptin given in combination with metformin as initial therapy improves glycaemic control in patients with type 2 diabetes compared with either monotherapy: a randomized controlled trial. Diabetes Obes Metab. 2009;11:611-22.

21. Pfützner A, Paz-Pacheco E, Allen E, Frederich R, Chen R. Initial combination therapy with saxagliptin and metformin provides sustained glycaemic control and is well tolerated for up to 76 weeks. Diabetes Obes Metab. 2011;13:567-76.
22. Williams-Herman D, Johnson J, Teng R, et al. Efficacy and safety of sitagliptin and metformin as initial combination therapy and as monotherapy over 2 years in patients with type 2 diabetes. Diabetes Obes Metab. 2010;12:442-51.

23. Williams-Herman D, Johnson J, Teng $\mathrm{R}$, et al. Efficacy and safety of initial combination therapy with sitagliptin and metformin in patients with type 2 diabetes: a 54-week study. Curr Med Res Opin. 2009;25:569-83.

24. Weissman P, Goldstein BJ, Rosenstock J, et al. Effects of rosiglitazone added to submaximal doses of metformin compared with dose escalation of metformin in type 2 diabetes: the EMPIRE Study. Curr Med Res Opin. 2005;21:2029-35.

25. Zinman B, Harris SB, Neuman J, et al. Low-dose combination therapy with rosiglitazone and metformin to prevent type 2 diabetes mellitus (CANOE trial): a double-blind randomised controlled study. Lancet. 2010;376:103-11.

26. Olansky L, Reasner C, Seck TL, et al. A treatment strategy implementing combination therapy with sitagliptin and metformin results in superior glycaemic control versus metformin monotherapy due to a low rate of addition of antihyperglycaemic agents. Diabetes Obes Metab. 2011;13:841-9.

27. Reasner C, Olansky L, Seck TL, et al. The effect of initial therapy with the fixed-dose combination of sitagliptin and metformin compared with metformin monotherapy in patients with type 2 diabetes mellitus. Diabetes Obes Metab. 2011;13:644-52. 